\title{
Quasi-micromechanical damage model for brittle solids with interacting microcracks
}

\author{
Xi-Qiao Feng ${ }^{a, *}$, Qing-Hua Qin ${ }^{b}$, Shou-Wen $\mathrm{Yu}^{\mathrm{a}}$ \\ a Department of Engineering Mechanics, Tsinghua University, Beijing 100084, China \\ ${ }^{\mathrm{b}}$ Centre for Advanced Materials Technology (CAMT), School of Aerospace, Mechanical and Mechatronic Engineering, \\ University of Sydney, Sydney, NSW 2006, Australia
}

Received 1 July 2002; received in revised form 21 January 2003

\begin{abstract}
The combination of phenomenological and micromechanical damage mechanics is a promising path to construct an applicable damage model with firm physical foundation. In this paper, a quasi-micromechanical model is presented for microcrack-weakened brittle or quasi-brittle materials. The microcracking damage is characterized in terms of the orientation domain of microcrack growth (DMG) as well as a scalar microcrack density parameter. The DMG describes the complex damage and its evolution associated with microcrack growth, while the scalar microcrack density factor defining the isotropic part of damage yields an easy calculation of the effects of microcrack interaction on effective elastic moduli. The conventional methods for estimating the effective moduli of microcracked solids, based on the concept of effective medium or effective field, are formulated in a universal framework. A novel and efficient scheme is suggested to calculate the microcrack interaction effects on the effective elastic moduli in a very convenient manner. Thereby, a quasi-micromechanical model is established to simulate the constitutive response of microcrack-weakened brittle or quasi-brittle materials under complex loading. Considering various micromechanisms of microcracking damage, the overall effective constitutive relation for different stages including linear elasticity, pre-peak non-linear hardening, stress drop and strain softening are expressed in a unified form.
\end{abstract}

(c) 2003 Elsevier Ltd. All rights reserved.

Keywords: Microcracking; Fracture mechanisms; Constitutive relation; Inhomogeneous material

\section{Introduction}

Nucleation, growth and coalescence of microcracks are typical damage mechanisms in brittle and quasi-brittle solids like concrete, ceramics, polycrystalline rocks and iron. Due to their

\footnotetext{
${ }^{*}$ Corresponding author. Tel.: +86-10-6277-2934; fax: +8610-6278-1824.

E-mail address: fengxq@tsinghua.edu.cn (X.-Q. Feng).
}

heterogeneous microstructures, these materials possess some complex and non-linear overall mechanical properties that are greatly different from those of ductile metals. Damage mechanics of brittle solids has been the subject of many investigations during the past three decades. Many phenomenological and micromechanical damage models have been established (see, e.g. Krajcinovic, 1989, 1997; Kachanov, 1994; Nemat-Nasser and Hori, 1993; Lacy et al., 1997; Lawn and Marshall, 1998; Feng and Yu, 2002 for review). In 
spite of the great development, some fundamental and important issues in this field have not been resolved yet. First, an internal variable is usually defined in continuum damage mechanics in the form of scalar, vector or tensor to describe the damage and its evolution in a solid. However, an internal variable with a small number of damage parameters is generally unable to describe the damage state exactly, while it is difficult to determine the evolution laws of damage parameters, especially when their number is large. To date, the description of damage is still a much argued problem (Krajcinovic, 1997; Krajcinovic and Mastilovic, 1995). Second, most of the previous damage models feel difficult to deal with problems of complex loadings. Such reasons as the non-association of damage evolution and the presence of stress drop and strain softening render the establishment of the overall effective constitutive relation of brittle materials very difficult. Third, the incorporation of microcrack-induced residual strains into the constitutive relation of brittle and quasi-brittle solids has attracted little attention, although both experimental observation and theoretical research have indicated its necessity (Najar, 1994; Feng and Gross, 2000). Fourth, the deformation and failure behaviors of brittle materials are very sensitive to the loading state, and may be greatly different under tension, compression and shearing.

In addition, how to calculate the effects of microcrack interaction on the overall constitutive relation is also an extensively argued but as yet unsettled issue. Estimates of the overall elastic moduli of a microcracked solid are usually obtained with the dilute-concentration method (DCM), self-consistent method (SCM) (Budiansky and O'Connell, 1976; Horii and Nemat-Nasser, 1983), differential method (DM) (Hashin, 1988), generalized self-consistent method (GSCM) (Aboudi and Benveniste, 1987; Huang et al., 1994), effective self-consistent method (Zheng and Du, 2001), and some other effective medium methods. The DCM, sometimes referred to as the Taylor model or the non-interacting approximation, completely neglects microcrack interaction and, therefore, is the simplest method. The SCM, DM and GSCM account for the microcrack interaction effects in different manners. However, they cannot ensure a good accuracy of the results and need cumbersome numerical calculation or very complicated derivation. Analytical results can be obtained only in a very few cases of microcrack distribution. For more general cases, these complicated estimation methods possess little attraction for application. When a brittle solid is subjected to complex loading, the evolution of microcracking damage is of tri-dimensional and complex anisotropy with the directions of its principal axes varying with loading. In this case, it is very difficult, if not impossible, to employ the SCM, DM and GSCM. Therefore, it is of significant interest to develop simpler methods to calculate the impacts of microcrack interaction on effective elastic properties.

To establish a relatively complete and applicable constitutive relation model, a rather novel and interesting path is to combine the methods and advantages of both phenomenological and micromechanical damage mechanics. To this end, two ways seem to be considerable. The first is to construct a model within the framework of continuum damage mechanics, while the definition of damage variable, the formulation of evolution law as well as the determination of constitutive parameters are specified on the basis of micromechanical consideration. Such a model may be referred to as a micromechanics-based phenomenological damage model, or a quasi-phenomenological damage model (Krajcinovic et al., 1991; Swoboda and Yang, 1999; Shao and Rudnicki, 2000). The second is to build the basic framework of the model directly from micromechanical analysis, while some concepts and skills of continuum damage mechanics are introduced to lead to a simple but more exact model with solid physical background. We refer to such a model as quasi-micromechanical. The well-known Gurson's model (Gurson, 1977) for ductile damage is just a classic example resulting from the combination of phenomenological and micromechanical analyses. To date, there is still a lack of a satisfactory damage theory of general applicability for microcracked brittle solids.

The purpose of the present work is to establish a quasi-micromechanical damage model for cal- 
culating the overall constitutive relation of brittle materials with interacting and evolutionary microcracks. The damage evolution is characterized in terms of orientation domain of microcrack growth (DMG) (Feng and Yu, 1995, 2002; Feng and Gross, 2000) as well as a scalar microcrack density parameter (Bristow, 1960; Budiansky and O'Connell, 1976). An outline of the paper is as follows. Section 2 brings the conventional estimation techniques of effective moduli of microcracked solids into a universal framework, and proposes a very simple and efficient scheme. Section 3 introduces the concept of DMG and the description of damage evolution. In Section 4, a quasi-micromechanical damage mechanics theory is presented on the basis of the DMG model to calculate the effects of microcrack interaction on the effective elastic constitutive relation using the method suggested in Section 2. Finally, an example of complex loading is given to illustrate the applicability of the presented model.

\section{Estimation methods for effective moduli}

\subsection{Universal framework}

Consider an elastic solid containing many distributed microcracks. To obtain the effective elastic moduli of random heterogeneous materials, one typically performs the ensemble-volume averaging process within a representative volume element (RVE) that satisfies two opposite requirements in size, that is, it is large enough to contain a large number, $N$ say, of microcracks and yet small enough to render its mapping on a continuum point meaningful. The boundary $\partial V$ of the RVE is subjected either to tractions in equilibrium with a uniform overall stress $\sigma^{\infty}$ or to displacements compatible to a prescribed uniform overall strain $\boldsymbol{\varepsilon}^{\infty}$, as shown in Fig. 1(a). Then, the effective compliance tensor $\mathbf{S}$ and stiffness tensor $\mathbf{L}$ are defined by

$\overline{\boldsymbol{\varepsilon}}=\mathbf{S}: \boldsymbol{\sigma}^{\infty}, \quad \overline{\boldsymbol{\sigma}}=\mathbf{L}: \boldsymbol{\varepsilon}^{\infty}$,

where $\overline{\boldsymbol{\varepsilon}}$ denotes the overall average strain over the $\mathrm{RVE}$ in the case of traction boundary condition, and $\overline{\boldsymbol{\sigma}}$ the overall average stress in the case of

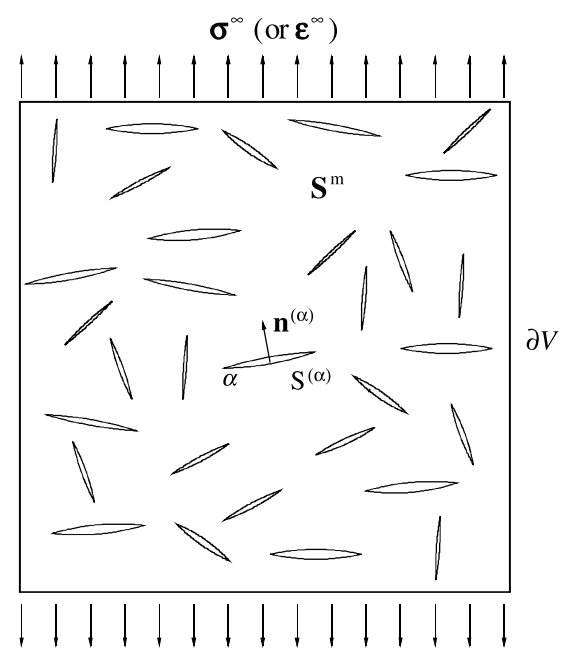

(a)

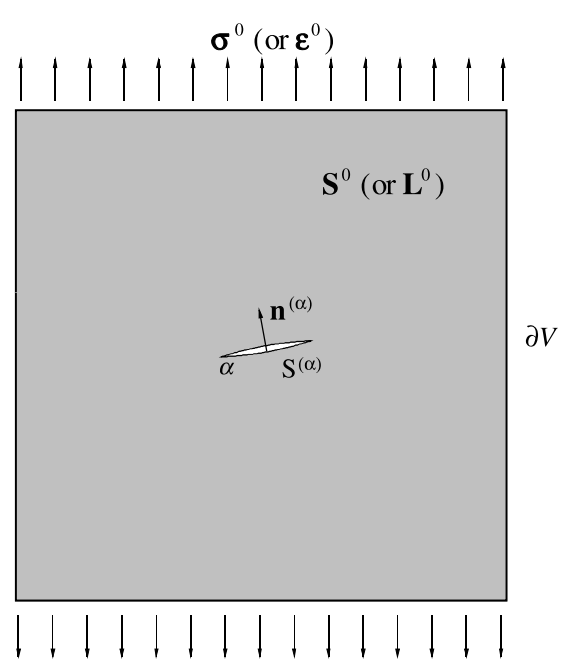

(b)

Fig. 1. (a) An RVE of a microcracked solid and (b) the simplified model for calculating the opening displacement of a microcrack.

displacement boundary condition. A colon between two tensors denotes contraction (inner production) over two indices. The average strain and stress can be decomposed as

$\overline{\boldsymbol{\varepsilon}}=\overline{\boldsymbol{\varepsilon}}^{\mathrm{m}}+\overline{\boldsymbol{\varepsilon}}^{\mathrm{c}}, \quad \overline{\boldsymbol{\sigma}}=\overline{\boldsymbol{\sigma}}^{\mathrm{m}}-\overline{\boldsymbol{\sigma}}^{\mathrm{c}}$,

where $\overline{\boldsymbol{\varepsilon}}^{\mathrm{m}}$ and $\overline{\boldsymbol{\sigma}}^{\mathrm{m}}$ denote the matrix strain and stress tensors averaged over the RVE, $\overline{\boldsymbol{\varepsilon}}^{\mathrm{c}}$ and $\overline{\boldsymbol{\sigma}}^{\mathrm{c}}$ denote the microcrack-induced variations in the 
overall average strain and stress tensors, respectively. The constitutive relation of the linear elastic matrix requires that

$\overline{\boldsymbol{\varepsilon}}^{\mathrm{m}}=\mathbf{S}^{\mathrm{m}}: \boldsymbol{\sigma}^{\infty}, \quad \overline{\boldsymbol{\sigma}}^{\mathrm{m}}=\mathbf{L}^{\mathrm{m}}: \boldsymbol{\varepsilon}^{\infty}$,

where $\mathbf{L}^{\mathrm{m}}$ and $\mathbf{S}^{\mathrm{m}}=\left(\mathbf{L}^{\mathrm{m}}\right)^{-1}$ are the stiffness and compliance of the matrix, respectively.

For simplicity in formulation, it is assumed that all microcracks are planar. In the case of traction boundary condition, the variation of the volumeaveraged strain due to the presence of microcracks can be calculated by

$\overline{\boldsymbol{\varepsilon}}^{\mathrm{c}}=\frac{1}{2 V} \sum_{\alpha=1}^{N} S^{(\alpha)}(\overline{\mathbf{b}} \mathbf{n}+\mathbf{n} \overline{\mathbf{b}})^{(\alpha)}$,

where the superscript $(\alpha)$ stands for a quantity of the $\alpha$ th microcrack, $S^{(\alpha)}, \overline{\mathbf{b}}^{(\alpha)}$ and $\mathbf{n}^{(\alpha)}$ denote the surface area, the average opening displacement discontinuity vector and the unit vector normal to the crack faces, respectively.

Thus, the key problem becomes how to calculate the opening displacement of a microcrack embedded in a solid containing many disordered microcracks. Evidently, it is very difficult to determine analytically the exact opening displacements due to the large number of interacting microcracks. Therefore, some simplifications or approximations are almost exclusively necessary. On one hand, the medium surrounding a microcrack is weakened by the neighboring microcracks, and then its stiffness is lower than the pristine matrix. On the other hand, the stress field around a microcrack is perturbed due to the existence of other microcracks. As a straightforward approximate model, thus, the microcrack is assumed to be surrounded by an effective medium, referred to also as the comparison or reference matrix, with compliance $\mathbf{S}^{0}$ (or stiffness $\mathbf{L}^{0}$ ) and subjected to an effective stress $\boldsymbol{\sigma}^{0}$ (or strain $\boldsymbol{\varepsilon}^{0}$ ) in the far field, as shown in Fig. 1(b). This approximation, which renders the analytical evaluation of effective moduli possible, is common to almost all the effective medium methods and the effective field methods, e.g. the DCM, SCM, DM and GSCM, though the definitions of $\mathbf{S}^{0}$ (or $\mathbf{L}^{0}$ ) and $\boldsymbol{\sigma}^{0}$ (or $\boldsymbol{\varepsilon}^{0}$ ) in them are different (Feng, 2001).

In the case of displacement boundary condition, the microcrack-induced decrease in the volume- averaged stress, $\overline{\boldsymbol{\sigma}}^{\mathrm{c}}$, can be written, similarly to (4), as

$\overline{\boldsymbol{\sigma}}^{\mathrm{c}}=\frac{1}{2 V} \mathbf{L}^{0}:\left[\sum_{\alpha=1}^{N} S^{(\alpha)}(\overline{\mathbf{b}} \mathbf{n}+\mathbf{n} \overline{\mathbf{b}})^{(\alpha)}\right]$.

In the approximate model in Fig. 1(b), the average opening displacement vector of a microcrack can be expressed as

$\overline{\mathbf{b}}=\mathbf{B}\left(\mathbf{S}^{0}, \mathscr{G}, \boldsymbol{\sigma}^{0}\right) \cdot \boldsymbol{\sigma}^{0} \cdot \mathbf{n}$,

in the case of traction boundary condition, or

$\overline{\mathbf{b}}=\mathbf{C}\left(\mathbf{L}^{0}, \mathscr{G}, \boldsymbol{\varepsilon}^{0}\right) \cdot \boldsymbol{\varepsilon}^{0} \cdot \mathbf{n}$,

in the case of displacement boundary condition. The second-rank symmetric tensor $\mathbf{B}$ in (6) is called the crack opening displacement tensor, and $\mathscr{G}$ signifies the geometry of the microcrack, and $\mathbf{C}=\mathbf{B}: \mathbf{L}^{0}$ is the crack opening displacement tensor in terms of $\mathbf{L}^{0}$ and $\boldsymbol{\varepsilon}^{0}$.

Define two fourth-order tensors $\mathbf{H}$ and $\mathbf{G}$ by

$\boldsymbol{\sigma}^{0}=\mathbf{H}: \boldsymbol{\sigma}^{\infty}, \quad \boldsymbol{\varepsilon}^{0}=\mathbf{G}: \boldsymbol{\varepsilon}^{\infty}$,

which relate the effective stress $\sigma^{0}$ to $\boldsymbol{\sigma}^{\infty}$, and the effective strain $\boldsymbol{\varepsilon}^{0}$ to $\boldsymbol{\varepsilon}^{\infty}$, respectively. Assume that the tensor $\mathbf{H}$ (or $\mathbf{G}$ ) is same for all microcracks, as is consistent with the inherent assumptions of the effective medium or effective field methods. Thus, all microcracks are subjected to the same far field $\boldsymbol{\sigma}^{0}$ (or $\varepsilon^{0}$ ), as shown in Fig. 1(b).

From Eqs. (4)-(8), the variations in the overall strain and stress induced by the $\alpha$ th single microcrack are obtained as

$$
\begin{aligned}
\overline{\boldsymbol{\varepsilon}}^{(\alpha)}= & \frac{S^{(\alpha)}}{4 V}\left[\boldsymbol{\sigma}^{0}:(\mathbf{n B n})+(\mathbf{B n}): \boldsymbol{\sigma}^{0} \mathbf{n}+(\mathbf{n B n}): \boldsymbol{\sigma}^{0}\right. \\
& \left.+\mathbf{n} \boldsymbol{\sigma}^{0}:(\mathbf{n B})\right]^{(\alpha)} \\
\overline{\boldsymbol{\sigma}}^{(\alpha)}= & \frac{S^{(\alpha)}}{4 V} \mathbf{L}^{0}:\left[\boldsymbol{\varepsilon}^{0}:(\mathbf{n C n})+(\mathbf{C n}): \boldsymbol{\varepsilon}^{0} \mathbf{n}\right. \\
& \left.+(\mathbf{n C n}): \boldsymbol{\varepsilon}^{0}+\mathbf{n} \boldsymbol{\varepsilon}^{0}:(\mathbf{n C})\right]^{(\alpha)},
\end{aligned}
$$

respectively. Thus, the overall compliance tensor $\mathbf{S}$ and stiffness tensor $\mathbf{L}$ of the microcracked solid are arrived at (Feng, 2001)

$$
\begin{aligned}
S_{i j k l}=S_{i j k l}^{\mathrm{m}} & +\frac{1}{4 V} H_{s t k l} \sum_{\alpha=1}^{N} S^{(\alpha)}\left(n_{i} B_{j s} n_{t}+n_{j} B_{i s} n_{t}\right. \\
& \left.+n_{i} B_{j t} n_{s}+n_{j} B_{i t} n_{s}\right)^{(\alpha)}
\end{aligned}
$$




$$
\begin{aligned}
L_{i j k l}=L_{i j k l}^{\mathrm{m}} & -\frac{1}{4 V} L_{i j m n}^{0} G_{s t k l} \sum_{\alpha=1}^{N} S^{(\alpha)}\left(n_{m} C_{n s} n_{t}\right. \\
& \left.+n_{n} C_{m s} n_{t}+n_{m} C_{n t} n_{s}+n_{n} C_{m t} n_{s}\right)^{(\alpha)},
\end{aligned}
$$

which possess the Voigt symmetry, i.e. $S_{i j k l}=$ $S_{j i k l}=S_{i j l k}=S_{k l i j} \quad$ and $\quad L_{i j k l}=L_{j i k l}=L_{i j l k}=L_{k l i j}$. Einstein's summation convention for the dummy indices is adopted herein and in the sequel.

\subsection{Effective mediums and effective fields}

Since the two cases of boundary condition in Fig. 1(b) can be discussed similarly, for conciseness, only the traction boundary condition is considered in what follows. Once the shapes and orientations of all microcracks (or their probability density functions) are specified, the overall stiffness or compliance tensor of the microcracked solid depends upon the tensors $\mathbf{B}$ and $\mathbf{H}$. Therefore, the interaction effects can be incorporated into the overall effective compliance tensor through an appropriate choice either of the comparison matrix $\mathbf{S}^{0}$ or of the far-field stress $\boldsymbol{\sigma}^{0}=\mathbf{H}: \boldsymbol{\sigma}^{\infty}$ in Fig. 1(b). Almost all the aforementioned estimation techniques based on an effective medium or effective field can be formulated in the form of Eqs. (11) and (12) and, in other words, have a complete formal similarity. Their differences stem only from the choices of $\mathbf{S}^{0}$ and $\boldsymbol{\sigma}^{0}$, i.e., from the "effective" environment where the microcrack is assumed to be embedded. In the DCM, for example, each microcrack is placed in the undamaged matrix $\left(\mathbf{S}^{0}=\mathbf{S}^{\mathrm{m}}\right)$ subjected to the unviolated remote stress $\boldsymbol{\sigma}^{0}=\boldsymbol{\sigma}^{\infty}$ (i.e., $\mathbf{H}=\mathbf{I}$ with I being the fourth-order identity tensor). In the SCM (Budiansky and O'Connell, 1976; Horii and Nemat-Nasser, 1983), the environment of each microcrack is the unviolated remote stress $\sigma^{0}=\sigma^{\infty}$ and a damaged effective medium having the as-yetunknown compliance $\left(\mathbf{S}^{0}=\mathbf{S}^{\mathrm{SCM}}\right)$.

In addition, it can be seen from Eqs. (6) and (11) that an effective field method may be mapped to an effective medium method in the sense that their estimates of effective moduli are identical. Substituting the first equation of (8) into (6) yields

$$
\overline{\mathbf{b}}=\left[\mathbf{B}\left(\mathbf{S}^{0}, \mathscr{G}, \boldsymbol{\sigma}^{0}\right) \cdot \mathbf{H}\right] \cdot \boldsymbol{\sigma}^{\infty} \cdot \mathbf{n} .
$$

Therefore, provided that one compliance tensor $\mathbf{S}^{\prime 0}$ can be found such that

$\mathbf{B}\left(\mathbf{S}^{\prime 0}, \mathscr{G}, \boldsymbol{\sigma}^{0}\right) \cdot \mathbf{I}=\mathbf{B}\left(\mathbf{S}^{\mathrm{m}}, \mathscr{G}, \boldsymbol{\sigma}^{0}\right) \cdot \mathbf{H}$,

then the effective medium method defined by $\mathbf{S}^{0}=\mathbf{S}^{\prime 0}$ and $\boldsymbol{\sigma}^{0}=\boldsymbol{\sigma}^{\infty}$ will lead to the same results as the effective field method defined by $\mathbf{S}^{0}=\mathbf{S}^{\mathrm{m}}$ and $\sigma^{0}$, that is to say, these two methods are equivalent with each other. This correspondence relation implies that one can construct an appropriate method for estimating the effects of microcrack interaction by changing only one of $\mathbf{S}^{0}$ and $\boldsymbol{\sigma}^{0}$ while keeping the other same as that in the noninteracting approximation.

\subsection{A new estimate scheme of effective moduli}

The arguments above suggest that apart from their heuristic foundations, the conventional methods for estimating the effective moduli are developed by only several possible choices of $\mathbf{S}^{0}$ and $\boldsymbol{\sigma}^{0}$, which are by no means better than others. Clearly, any number of other choices is admissible for this purpose. The relevant necessary conditions that should be met by such $\mathbf{S}^{0}$ and $\boldsymbol{\sigma}^{0}$ are as follows (Feng, 2001):

(1) The comparison matrix compliance $\mathbf{S}^{0}$ should be limited by a lower bound $\mathbf{S}^{-}$and an upper bound $\mathbf{S}^{+}$, that is, $\left(\mathbf{S}^{+}-\mathbf{S}^{0}\right)$ and $\left(\mathbf{S}^{0}-\mathbf{S}^{-}\right)$are required to be positive self-definite. For example, the pristine matrix compliance $\mathbf{S}^{\mathrm{m}}$ or the DCM compliance $\mathbf{S}^{\mathrm{DCM}}$ may be adopted as a lower bound of $\mathbf{S}^{0}$, and the SCM compliance $\mathbf{S}^{\mathrm{SCM}}$ may be used as an upper bound.

(2) Generally, the stress amplifying effect plays a more significant role than the stress shielding effect in the effective moduli of microcracked solids. Therefore, $\sigma^{0}$ should be limited by a lower bound $\boldsymbol{\sigma}^{\infty}$.

In addition, three further conditions are required to yield an applicable method. First, the effective medium $\mathbf{S}^{0}$ and the effective stress $\boldsymbol{\sigma}^{0}$ can be determined readily. Second, the calculation of the overall effective moduli should be relatively easy. In these two senses, the SCM, DM and GSCM are not appropriate. Third, the results 
obtained should be of satisfactory accuracy for general cases of microcrack arrays.

Subject to the above conditions, we choose (Feng, 2001)

$\boldsymbol{\sigma}^{0}=\boldsymbol{\sigma}^{\infty}, \quad \mathbf{S}^{0}=\left(1-\xi f^{\eta}\right)^{-1} \mathbf{S}^{\mathrm{m}}$,

where $\xi$ and $\eta$ are two adjustable parameters, and $f$ is the conventional scalar microcrack density parameter defined as (Bristow, 1960; Budiansky and O'Connell, 1976)

$f=\frac{2}{\pi} \sum_{\alpha=1}^{N} \frac{\left(S^{(\alpha)}\right)^{2}}{P^{(\alpha)}}$

where $P^{(\alpha)}$ is the perimeter of the $\alpha$ th crack.

The effective medium method defined by Eq. (15) is first used to consider, for example, an isotropic solid containing penny shaped microcracks of completely random orientations and locations. In this case, the results of DCM, SCM and some other approximate methods are available in the references but omitted here for shortness. For an open circular crack normal to the $x_{2}$-axis in an isotropic matrix with Young's modulus $E_{0}$ and Poisson's ratio $v_{0}$, for instance, the non-zero components of $\mathbf{B}$ in the local coordinate system are (Sneddon, 1951)

$B_{11}^{\prime}=B_{33}^{\prime}=\frac{16\left(1-v_{0}^{2}\right)}{\pi E_{0}\left(2-v_{0}\right)}, \quad B_{22}^{\prime}=\frac{8\left(1-v_{0}^{2}\right)}{\pi E_{0}}$.

From Eqs. (11), (15) and (17), the normalized effective Young's modulus and shear modulus are obtained very easily as

$$
\begin{aligned}
& \frac{E}{E^{\mathrm{m}}}=\left[1+\frac{16\left(1-\left(v^{\mathrm{m}}\right)^{2}\right)\left(10-3 v^{\mathrm{m}}\right) f}{45\left(2-v^{\mathrm{m}}\right)\left(1-\xi f^{\eta}\right)}\right]^{-1}, \\
& \frac{G}{G^{\mathrm{m}}}=\left[1+\frac{32\left(1-v^{\mathrm{m}}\right)\left(5-v^{\mathrm{m}}\right) f}{45\left(2-v^{\mathrm{m}}\right)\left(1-\xi f^{\eta}\right)}\right]^{-1},
\end{aligned}
$$

where $E^{\mathrm{m}}, G^{\mathrm{m}}$ and $v^{\mathrm{m}}$ are the Young's modulus, shear modulus and Poisson's ratio of the pristine matrix. It is seen that the new estimation scheme defined by (15) has the following advantages. First, the derivation of the effective moduli is as straightforward and easy as the DCM, without numerical iteration being required. Second, the choice of (15) can yield the results in good agreement with DCM, SCM, GSCM, DM when taking appropriate values of $\xi$ and $\eta$. For example, the results (18) of $\xi=0$ reduce to those obtained from the DCM. When $\xi=1.778$ and $\eta=1.0$, the results are in a good agreement with the SCM estimates. For application, the values of the constants $\xi$ and $\eta$ can be determined by fitting experimental results or other theoretical results of good accuracy. By comparing the results of the present method and the GSCM for the two extreme cases of isotropy and complete anisotropy, we suggest that $\xi=4 / 9$ and $\eta=1.0$. The resulted method leads to effective elastic moduli of satisfactory accuracy for general arrangements of microcracks, as can be verified by comparing with numerical examples (Feng et al., 2003), experimental measurements (Carvalho and Labuz, 1996) and other analytical methods of good accuracy (Zheng and Du, 2001). Though only two scalar parameters are used in Eq. (15), in addition, this method is appropriate not only for isotropic damage but also for anisotropic damage. It is easy to understand that the modification magnitudes to the effective elastic moduli due to microcrack interaction are direction-dependent.

\section{Quasi-micromechanical damage model}

\subsection{DMG and its evolution}

Assume that the number density of microcracks in a brittle material is $n_{\mathrm{c}}$, and that in the initial undamaged state all microcracks have the same statistically averaged radius, $a_{0}$. Such failure-related properties as the tensile strength and the strain softening behavior are sensitive to the concrete sizes, locations and orientations of individual microcracks, namely, higher-order effects of microcrack interaction (Feng and $\mathrm{Yu}, 2002$ ). However, such transport properties as the effective elastic moduli and thermal conductivities of microcracked solids are related merely to the statistically averaged parameters of microcrack distribution. Therefore, we adopt the assumption that all microcracks have the same average size at the initial state, though it is easy to incorporate any other statistical distribution rules of microcrack sizes into the present model. First, consider a single microcrack in the RVE. Refer to a global 


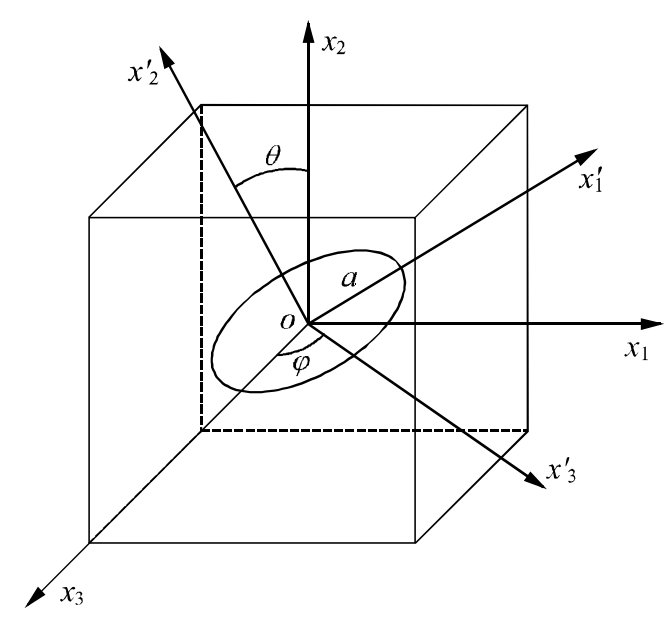

Fig. 2. A microcrack with global $\left(x_{i}\right)$ and local $\left(x_{i}^{\prime}\right)$ coordinate systems.

Cartesian coordinate system $\left(o-x_{1} x_{2} x_{3}\right)$ and a local Cartesian coordinate system $\left(o-x_{1}^{\prime} x_{2}^{\prime} x_{3}^{\prime}\right)$ for the $\alpha$ th microcrack, as shown in Fig. 2, in which the $x_{2}^{\prime}-$ axis is parallel to the normal $\mathbf{n}$ of the microcrack, and the $x_{3}^{\prime}$-axis is coplanar with $x_{1}$ and $x_{3}$. Then the orientation of the microcrack is expressible in terms of two angles, $(\theta, \varphi)$, as defined in Fig. 2.

For practical applications, assume that an open microcrack under tension will grow in a self-similar fashion when the average value of the strain energy release rate along the microcrack edge reaches a critical value. Then the mixed-mode fracture criterion for a penny shaped microcrack in an isotropic medium may be expressed as (see, e.g. Kanninen and Popelar, 1985; Ju and Lee, 1991; Feng and $\mathrm{Yu}, 1995)$

$$
\left(\frac{K_{\mathrm{I}}^{\prime}}{K_{\mathrm{IC}}}\right)^{2}+\left(\frac{K_{\mathrm{II}}^{\prime}}{K_{\mathrm{IIC}}}\right)^{2}=1,
$$

where $K_{\mathrm{I}}^{\prime}$ and $K_{\mathrm{II}}^{\prime}$ represent the mode I and II SIFs, $K_{\mathrm{IC}}$ and $K_{\mathrm{IIC}}$ their intrinsic critical values, respectively. $K_{\mathrm{I}}^{\prime}$ and $K_{\mathrm{II}}^{\prime}$ are defined by

$$
\begin{aligned}
& K_{\mathrm{I}}^{\prime}=2 \sigma_{22}^{\prime} \sqrt{\frac{a}{\pi}} \\
& K_{\mathrm{II}}^{\prime}=\frac{4}{2-\pi} \sqrt{\frac{a}{\pi}\left[\left(\sigma_{21}^{\prime}\right)^{2}+\left(\sigma_{23}^{\prime}\right)^{2}\right]},
\end{aligned}
$$

where the stress tensor in the local coordinate system, $\sigma_{i j}^{\prime}$, is related to that in the global coordinate system, $\sigma_{i j}$, via the relation

$\sigma_{i j}^{\prime}=g_{i k}^{\prime} g_{j l}^{\prime} \sigma_{k l}$,

where the transformation matrix $g_{i j}^{\prime}$ is given by

$g_{i j}^{\prime}=\left[\begin{array}{ccc}\cos \theta \cos \varphi & \sin \theta & -\cos \theta \sin \varphi \\ -\sin \theta \cos \varphi & \cos \theta & \sin \theta \sin \varphi \\ \sin \varphi & 0 & \cos \varphi\end{array}\right]$.

As aforementioned, the growth of a microcrack is sensitive to its direct interaction with other microcracks, which depends upon the exact positions, sizes and orientations of microcracks of a large number. Such a problem can be solved only by some simplified numerical methods (Feng et al., 2003, and references therein). For simplicity, therefore, we do not address the interaction effects on microcrack growth in the fracture criterion (19).

Once a microcrack satisfies the criterion (19), it will propagate increasing its radius from $a_{0}$ to a certain characteristic value $a_{\mathrm{u}}$ and then be arrested by energy barriers (such as phase boundaries of different directions) with higher strength than interfaces. The same assumption of high-energy barriers that serve as a microcrack trapping mechanism was adopted by Krajcinovic and Fanella (1986), Ju and Lee (1991) and some others. The radius $a_{\mathrm{u}}$ depends on the microstructures of the material, such as sizes and shapes of grains or aggregates. Because the contribution of a microcrack into the effective compliance is proportional to the third power of its radius, the average radii $a_{0}$ and $a_{\mathrm{u}}$ are defined by

$a_{0}^{3}=\frac{1}{N_{0}} \sum_{\alpha=1}^{N_{0}}\left(a_{0}^{(\alpha)}\right)^{3}, \quad a_{\mathrm{u}}^{3}=\frac{1}{N_{\mathrm{u}}} \sum_{\alpha=1}^{N_{\mathrm{u}}}\left(a_{\mathrm{u}}^{(\alpha)}\right)^{3}$,

where $N_{0}$ is the total number of microcracks in the RVE at the initial undamaged state, and $N_{\mathrm{u}}$ the number of microcracks that have propagated.

Thus, the microcracking damage state can be characterized by the concept of DMG, which is defined as the possible orientation scope in the orientation space $(\theta, \varphi)$ of all microcracks that have propagated in the aforementioned fashion ( $\mathrm{Yu}$ and Feng, 1995). In other words, all microcracks 


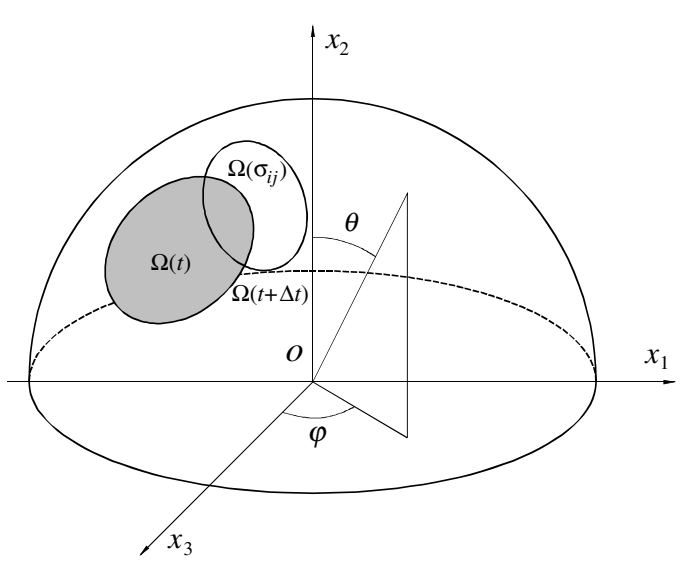

Fig. 3. Evolution of a DMG $\Omega(t)$ with loading in the orientation space $(\theta, \varphi)$.

whose orientations are within the orientation scope of DMG must have propagated and have the radius $a_{\mathrm{u}}$. The DMG can be shown in the upper half of the unit sphere surface (Fig. 3), since $\theta$ and $\varphi$ take their values within the ranges $[0, \pi / 2]$ and $[0,2 \pi]$, respectively. As the applied stresses vary with time $t$, more and more microcracks may satisfy the fracture criterion (19) and so the DMG of the material, $\Omega(t)$, may evolve with time. Since the propagation of microcracks is irreversible, the evolution of DMG is irreversible too, that is,

$\Omega(t+\Delta t) \supseteq \Omega(t)$.

The evolution equation of DMG with loading is expressed by the summation of sets as (Fig. 3)

$\Omega(t+\Delta t)=\Omega(t) \cup \Omega\left(\sigma_{i j}(t+\Delta t)\right)$,

where $\Omega\left(\sigma_{i j}\right)$ denotes the DMG corresponding to the stress tensor $\sigma_{i j}$ under the condition of monotonically proportional loading. The detailed formulas for calculating $\Omega\left(\sigma_{i j}\right)$ were given in $\mathrm{Yu}$ and Feng (1995). The DMG defined above includes few material parameters, and its evolution with damage progress can be easily formulated by using the calculation rules of sets. The basic idea of DMG is to divide all the microcracks into some groups or sets according to their different deformation and growth behaviors and then to incorporate the contributions of various damage mechanisms in the constitutive relation respectively. Due to the special geometry feature of mi- crocracks, their deformation and propagation are closely related to their orientations. Therefore, the DMG provides a heuristic and convenient approach for describing the evolving damage due to distributed microcracks.

With further increase in applied stresses, some microcracks normal or nearly normal to the maximum principal tensile stress may pass through the high-energy barriers and experience the secondary growth. Similar to (19), the criterion of secondary growth of a circular microcrack may take the following form (Feng and $\mathrm{Yu}, 1995$ )

$\left(\frac{K_{\mathrm{I}}^{\prime}}{K_{\mathrm{ICC}}}\right)^{2}+\left(\frac{K_{\mathrm{II}}^{\prime}}{K_{\mathrm{IICC}}}\right)^{2}=1$

where $K_{\text {ICC }}$ and $K_{\text {IICC }}$ are respectively the critical values of mode I and II SIFs of energy barriers, often taken as the values of fracture toughness of the pristine matrix. When some microcracks have experienced the secondary growth, the damage and deformation will generally be localized in the material causing the rapid stress drop and strain softening phenomena in the stress-strain curve. The secondary growth of a microcrack generally corresponds to a traversing of microcrack propagation in different microstructures (e.g., the transition from an interface crack to a transgranular crack). The microscopic damage mechanisms, the overall constitutive relation as well as the residual strains during the stages subsequent to the occurrence of stress drop have been studied in detail by Feng and Yu (1995, 2002), and Feng and Gross (2000).

\subsection{Quasi-micromechanical DMG model}

As aforementioned, the DCM is very convenient for providing an estimate of effective elastic stiffness, but its evident, intrinsic shortcoming is the complete neglect of microcrack interaction. On the other hand, the SCM, DM and other effective medium approaches enable the implementation of the weak microcrack interaction effects in effective moduli, but the prohibitive complexity and the cumbersome computation limit their application, especially in the case of evolutionary damage. Under a prescribed loading path, the damage state of a brittle material may be very complex. Al- 
though it can be proved that all damaged materials with planar microcracks are of orthotropic anisotropy (Kachanov, 1994), the principal axes of anisotropy may evolve depending upon the loading path. Such problems are too complicated to be solved with such traditional methods as SCM and DM. Just for this reason, very little work has been done in the field of micromechanics to consider the calculation of overall constitutive relation of materials with microstructural evolution under complex loading, in spite of that some phenomenological models can be found in the literature (e.g. Ortiz, 1985).

As argued in the introduction, a promising path to achieve this aim is to combine the phenomenological and the micromechanical damage mechanics. Such an attempt is made here to present a quasi-micromechanical damage model. Besides the concept of DMG, the scalar microcrack density parameter $f$ defined in (16) is adopted to describe the damage of a microcracked solid. The DMG functions as an exact descriptor of the anisotropic microcracking damage state, while $f$, which defines merely the magnitude of the isotropic part of damage, is introduced to render the calculation of effective moduli much easier. Thereby, the new estimation scheme proposed in Section 2.3 can be implemented into the micromechanics-based DMG damage model. The microcrack density parameter $f(t)$ is related to the DMG $\Omega(t)$ by

$$
\begin{aligned}
f(t)= & n_{\mathrm{c}}[\bar{a}(t)]^{3} \\
= & n_{\mathrm{c}} a_{0}^{3}+n_{\mathrm{c}} \iint_{\Omega(t)} p(a, \theta, \varphi)\left(a_{\mathrm{u}}^{3}-a_{0}^{3}\right) \\
& \times \sin \theta \mathrm{d} \theta \mathrm{d} \varphi,
\end{aligned}
$$

where $p(a, \theta, \varphi)$ denotes the probability density function describing the distribution of the orientations and sizes of microcracks in the material.

Then, the effective compliance tensor of the damage solid can be estimated by embedding each microcrack into an effective medium with compliance $\mathbf{S}^{0}$ defined in (15) and subjected to the farfield stress $\boldsymbol{\sigma}^{\infty}$. The non-zero components of the crack opening displacement discontinuity vector in (17) become

$$
\begin{aligned}
& B_{11}^{\prime}=B_{33}^{\prime}=\frac{16\left(1-v^{2}\right)}{\pi E(2-v)(1-4 f / 9)}, \\
& B_{22}^{\prime}=\frac{8\left(1-v^{2}\right)}{\pi E(1-4 f / 9)} .
\end{aligned}
$$

Thus, the compliance tensor induced by the elastic deformation of the $\alpha$ th microcrack of radius $a$ and orientation $(\theta, \varphi)$ can be written as

$$
\begin{aligned}
\bar{S}_{i j k l}^{\mathrm{c}(\alpha)}(a, \theta, \varphi, \boldsymbol{\sigma})= & \frac{\pi a^{3}}{6} B_{m n}^{\prime}\left(g_{m i}^{\prime} n_{j}+g_{m j}^{\prime} n_{i}\right)\left(g_{2 k}^{\prime} g_{n l}^{\prime}\right. \\
& \left.+g_{2 l}^{\prime} g_{n k}^{\prime}\right),
\end{aligned}
$$

which is similar to that of the non-interacting approximation (Feng and Gross, 2000) except the difference in the values of $B_{m n}^{\prime}$. In what follows, the compliance tensor induced by the elastic deformation of a single microcrack is simply denoted as $\bar{S}_{i j k l}^{\mathrm{c}}(a)$.

The above discuss implies that the suggested scheme combines the advantages of both the DCM and the first-order effective medium methods. It considers the effects of microcrack interaction on the constitutive relation in a manner as convenient as the DCM. As mentioned in Section 2.3, in addition, the results obtained from the present method are expected to be better than those from the SCM, which overestimates the effects of microcrack interaction.

Evidently, the hypothesized effective medium defined in (15) is anisotropic provided that the pristine matrix is anisotropic. If the pristine matrix is isotropic, on the other hand, the suggested method calculates the opening displacement of a microcrack by assuming that it is embedded in an isotropic, infinite effective medium, as is similar to the Taylor's model. Their difference is that the effective medium in the present method is, instead of the pristine matrix, an approximate effective medium with degraded effective elastic moduli depending upon the actual damage state.

In the case that all microcracks are uniformly distributed in the orientation space, i.e.,

$p(a, \theta, \varphi)=\frac{1}{2 \pi}$,

the microcrack density $f$ in (27) is recast as

$$
\begin{aligned}
f & =n_{\mathrm{c}}[\bar{a}(t)]^{3} \\
& =n_{\mathrm{c}} a_{0}^{3}+\frac{n_{\mathrm{c}}}{2 \pi}\left(a_{\mathrm{u}}^{3}-a_{0}^{3}\right) \iint_{\Omega(t)} \sin \theta \mathrm{d} \theta \mathrm{d} \varphi .
\end{aligned}
$$


It is immediately evident that $f=n_{\mathrm{c}} a_{0}^{3}$ at the initial state, and $f=n_{\mathrm{c}} a_{\mathrm{u}}^{3}$ when all microcracks have experienced the first growth. The integral in (31) represents the area of the DMG $\Omega(t)$ in the upper half of the unit sphere surface, as shown in Fig. 3.

\subsection{Constitutive relation}

In this paper, only open microcracks are considered. If the stress component normal to a microcrack is compressive, assume that the effective shear stress between the crack faces is smaller than the maximum friction stress and no frictional sliding occurs, that is, all closed microcracks are assumed to be inactive. The DMG model including the complex mechanisms of closed microcracks under compression can be found in $\mathrm{Yu}$ and Feng (1995).

Considering the various mechanisms of microcracking damage, the overall effective constitutive relation for all the four stages including linear elasticity, pre-peak non-linear hardening, stress drop and strain softening can be expressed in the following unified form

$\varepsilon_{i j}=\left[S_{i j k l}^{0}+S_{i j k l}^{\mathrm{c} 1}+S_{i j k l}^{\mathrm{c} 2}+S_{i j k l}^{\mathrm{c} 3}\right] \sigma_{k l}+\varepsilon_{i j}^{\mathrm{R}}$,

where $S_{i j k l}^{\mathrm{c} 1}, S_{i j k l}^{\mathrm{c} 2}, S_{i j k l}^{\mathrm{c} 3}$ denote the increments of the compliance tensor due to microcracks that have not propagated $\left(a=a_{0}\right)$, that have experienced the first growth $\left(a=a_{\mathrm{u}}\right)$ and that have undergone the secondary growth $\left(a>a_{\mathrm{u}}\right)$, respectively. $\varepsilon_{i j}^{\mathrm{R}}$ denotes the strains due to the residual deformation of microcracks. We have

$$
\begin{aligned}
S_{i j k l}^{\mathrm{c} 1}= & \int_{0}^{2 \pi} \int_{0}^{\pi / 2} n_{\mathrm{c}} p(a, \theta, \varphi) \bar{S}_{i j k l}^{\mathrm{c}}\left(a_{0}\right) \sin \theta \mathrm{d} \theta \mathrm{d} \varphi \\
& -\iint_{\Omega} n_{\mathrm{c}} p(a, \theta, \varphi) \bar{S}_{i j k l}^{\mathrm{c}}\left(a_{0}\right) \sin \theta \mathrm{d} \theta \mathrm{d} \varphi, \\
S_{i j k l}^{\mathrm{c} 2}= & \iint_{\Omega} n_{\mathrm{c}} p(a, \theta, \varphi) \bar{S}_{i j k l}^{\mathrm{c}}\left(a_{\mathrm{u}}\right) \sin \theta \mathrm{d} \theta \mathrm{d} \varphi \\
& -\int_{0}^{2 \pi} \int_{0}^{\theta_{\mathrm{cc}}} n_{\mathrm{c}} p(a, \theta, \varphi) \bar{S}_{i j k l}^{\mathrm{c}}\left(a_{\mathrm{u}}\right) \sin \theta \mathrm{d} \theta \mathrm{d} \varphi, \\
S_{i j k l}^{\mathrm{c} 3}= & \int_{0}^{2 \pi} \int_{0}^{\theta_{\mathrm{cc}}} n_{\mathrm{c}} p(a, \theta, \varphi) \bar{S}_{i j k l}^{\mathrm{c}}\left(a_{\mathrm{s}}\right) \sin \theta \mathrm{d} \theta \mathrm{d} \varphi,
\end{aligned}
$$

$$
\begin{aligned}
\varepsilon_{i j}^{\mathrm{R}}= & \iint_{\Omega} n_{\mathrm{c}} p(a, \theta, \varphi) \bar{\varepsilon}_{i j}^{\mathrm{R}}\left(a_{\mathrm{u}}\right) \sin \theta \mathrm{d} \theta \mathrm{d} \varphi \\
+ & \int_{0}^{2 \pi} \int_{0}^{\theta_{\mathrm{cc}}} n_{\mathrm{c}} p(a, \theta, \varphi)\left[\bar{\varepsilon}_{i j}^{\mathrm{R}}\left(a_{\mathrm{s}}\right)\right. \\
& \left.-\bar{\varepsilon}_{i j}^{\mathrm{R}}\left(a_{\mathrm{u}}\right)\right] \sin \theta \mathrm{d} \theta \mathrm{d} \varphi,
\end{aligned}
$$

where $\theta_{\text {cc }}$ is an angle parameter related to the number of microcracked that have experienced the secondary growth (Feng and $\mathrm{Yu}, 1995), \bar{\varepsilon}_{i j}^{\mathrm{R}}(a)$ the residual strains induced by a microcrack of radius $a$, and $a_{\mathrm{s}}$ the radius of a microcrack during the secondary growth.

\section{Illustration}

Assume that all microcracks are distributed uniformly in the orientation space. In general cases of complex loading, the overall constitutive relation of brittle materials weakened by microcracks can be calculated from the present model with the aid of a simple computer program, because its derivation is often tedious, though straightforward. In this section, we consider an example of complex loading as shown in Fig. 4. The specimen is subjected to a uniaxial tensile stress $\sigma$ with $\sigma_{\mathrm{c}} \leqslant \sigma \leqslant \sigma_{\mathrm{cc}}$ in the direction of $\pi / 4$ measured from the $x_{1}$ - or $x_{2}$-axis, and rotates with respect to the $x_{2}$-axis. During the first cycle of rotation, more and more microcracks propagate and the DMG evolves. However, no microcrack will grow during

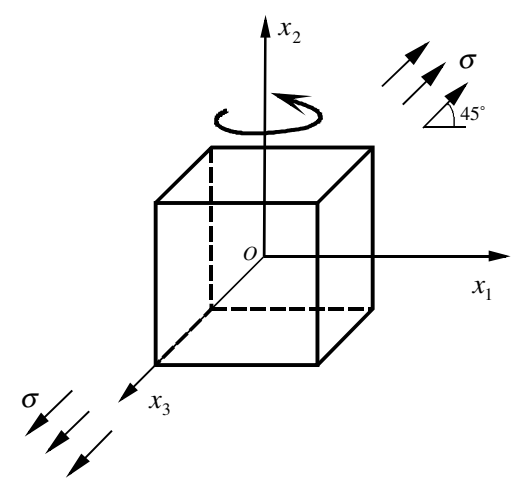

Fig. 4. A rotating brittle solid under uniaxial tension in the $45^{\circ}$ direction. 
the subsequent cycles because the fatigue growth of microcracks is disregarded here. The analytical solution of this example can be derived easily by using the present model. For short, only the damage and the stress-strain relation after the first circle of rotation are given in what follows. The DMG is expressed as

$\Omega(\sigma)=\left\{\theta_{1}(\sigma) \leqslant \theta \leqslant \theta_{2}(\sigma), 0 \leqslant \varphi \leqslant 2 \pi\right\}$,

where $\quad \theta_{1}(\sigma)=\pi / 4-\theta_{\max }(\sigma), \quad \theta_{2}(\sigma)=\pi / 4+$ $\theta_{\max }(\sigma)$, and $\theta_{\max }(\sigma)$ is a function of the tensile stress defined by

$\tan ^{2} \theta_{\max }(\sigma)=\frac{B_{2}-\sqrt{B_{2}^{2}-4 B_{1} B_{3}}}{B_{1}}$,

$B_{1}=-\frac{\pi}{4 a_{0}} K_{\mathrm{IIC}}^{2}, \quad B_{2}=-\frac{\pi}{2 a_{0}} K_{\mathrm{IIC}}^{2}-\left(\frac{2 \sigma}{2-v}\right)^{2}$,

$B_{3}=\left(\frac{\sigma K_{\mathrm{IIC}}}{K_{\mathrm{IC}}}\right)^{2}-\frac{\pi}{4 a_{0}} K_{\mathrm{IIC}}^{2}$.

The corresponding stress-strain relation can be obtained readily by substituting the DMG in (35) into (32). Some components of the elastic compliance tensor are given as

$$
\begin{aligned}
S_{2222}= & \frac{1}{E}+\frac{\rho(10-3 v) a_{0}^{3}}{E^{\prime}}+\frac{\rho\left(a_{\mathrm{u}}^{3}-a_{0}^{3}\right)}{E^{\prime}} \\
& \times\left[F_{1}\left(\theta_{2}\right)-F_{1}\left(\theta_{1}\right)\right], \\
S_{1111}= & \frac{1}{E}+\frac{\rho(10-3 v) a_{0}^{3}}{E^{\prime}}+\frac{\rho\left(a_{\mathrm{u}}^{3}-a_{0}^{3}\right)}{E^{\prime}} \\
& \times\left[F_{2}\left(\theta_{2}\right)-F_{2}\left(\theta_{1}\right)\right], \\
S_{1122}= & -\frac{v}{E}-\frac{\rho v a_{0}^{3}}{E^{\prime}}-\frac{\rho v\left(a_{\mathrm{u}}^{3}-a_{0}^{3}\right)}{2 E^{\prime}}\left[F_{3}\left(\theta_{2}\right)-F_{3}\left(\theta_{1}\right)\right], \\
S_{1133}= & -\frac{v}{E}-\frac{\rho v a_{0}^{3}}{E^{\prime}}-\frac{\rho v\left(a_{\mathrm{u}}^{3}-a_{0}^{3}\right)}{8 E^{\prime}}\left[F_{4}\left(\theta_{2}\right)-F_{4}\left(\theta_{1}\right)\right], \\
S_{1212}= & \frac{2(1+v)}{E}+\frac{\rho(5-v) a_{0}^{3}}{E^{\prime}} \\
& +\frac{15 \rho\left(a_{\mathrm{u}}^{3}-a_{0}^{3}\right)}{2 E^{\prime}}\left[F_{5}\left(\theta_{2}\right)-F_{5}\left(\theta_{1}\right)\right], \\
S_{1313}= & \frac{2(1+v)}{E}+\frac{\rho(5-v) a_{0}^{3}}{E^{\prime}} \\
& +\frac{15 \rho\left(a_{\mathrm{u}}^{3}-a_{0}^{3}\right)}{2 E^{\prime}}\left[F_{6}\left(\theta_{2}\right)-F_{6}\left(\theta_{1}\right)\right],
\end{aligned}
$$

with

$$
\begin{aligned}
\left.\rho=\frac{16}{45(2-v)}, \quad v^{2}\right) n_{\mathrm{c}} & =\frac{E}{(1-4 f / 9)} \\
F_{1}(\theta)= & 6 \cos ^{5} \theta-10 \cos ^{3} \theta+3(2-v)\left(1-5 \cos ^{5} \theta\right), \\
F_{2}(\theta)= & 5\left(2-3 \cos \theta+\cos ^{3} \theta\right) \\
& -\frac{3 v}{8}\left(8-15 \cos \theta+10 \cos ^{3} \theta-3 \cos ^{5} \theta\right) \\
F_{3}(\theta)= & 3 \cos ^{5} \theta-5 \cos ^{3} \theta \\
F_{4}(\theta)= & 8-15 \cos \theta+10 \cos ^{3} \theta-3 \cos ^{5} \theta \\
F_{5}(\theta)= & \frac{2}{3}-\frac{1}{2} \cos \theta-\frac{1}{6} \cos ^{3} \theta \\
& -v\left(\frac{2}{15}-\frac{1}{3} \cos ^{3} \theta+\frac{1}{5} \cos ^{5} \theta\right) \\
& 2 \\
F_{5}(\theta)= & \frac{\cos \theta+\frac{1}{3} \cos ^{3} \theta}{}-\frac{v}{4}\left(\frac{8}{15}-\cos \theta+\frac{1}{3} \cos ^{3} \theta-\frac{1}{5} \cos ^{5} \theta\right)
\end{aligned}
$$

All the other components of $\mathbf{S}$ can be obtained from its three properties: (1) the Voigt symmetry, i.e. $S_{i j k l}=S_{j i k l}=S_{i j l k}=S_{k l i j}$, (2) $S_{i j k l}=0$ whenever a subscript appears an odd number of times, and (3) the transversal isotropy with respect to the $x_{2}$ axis. The above solution of the effective compliance is reduced to the non-interacting or DCM solution by replacing $E^{\prime}$ with $E$.

The residual strains after the first cycle are given as

$$
\begin{aligned}
\bar{\varepsilon}_{22}^{\mathrm{R}}= & \frac{1}{3} \pi n_{\mathrm{c}} a_{\mathrm{u}}^{2} \bar{b}^{\mathrm{R}}\left(a_{\mathrm{u}}\right)\left(\cos ^{3} \theta_{1}-\cos ^{3} \theta_{2}\right), \\
\bar{\varepsilon}_{11}^{\mathrm{R}}= & \frac{1}{6} \pi n_{\mathrm{c}} a_{\mathrm{u}}^{2} \bar{b}^{\mathrm{R}}\left(a_{\mathrm{u}}\right)\left(\cos ^{3} \theta_{2}-3 \cos \theta_{2}\right. \\
& \left.\quad-\cos ^{3} \theta_{1}+3 \cos \theta_{1}\right),
\end{aligned}
$$

where $\bar{b}^{\mathrm{R}}\left(a_{\mathrm{u}}\right)$ denotes the average residual opening displacement of a microcrack of radius $a_{\mathrm{u}}$ and is formulated in Feng and Gross (2000).

More examples illustrating the application of the present model are omitted here. It is seen that the quasi-micromechanical model can yield relatively easily the constitutive relation with microcrack interaction for quasi-brittle materials under complex loading. 


\section{Conclusions}

Most of the previous damage models can be divided into two classes, phenomenological and micromechanical. A phenomenological one reflects the damage effects in terms of an internal variable of scalar, vector or tensor form, without deep research on physical mechanisms. Such a model usually possesses a relatively simple framework, but cannot explain some complicated phenomena associated with microcracking evolution due to its unsubstantial physical foundation. On the other hand, a micromechanical model can provide an insight into some failure behaviors of materials. Due to the prohibitive complexity of micromechanisms and the numerous factors influencing damage process, however, a series of simplifying assumptions are usually made in such a model yielding the results quantitatively unsatisfactory. Quasi-phenomenological or quasi-micromechanical models that combine the concepts and methods of the two kinds of conventional damage mechanics seem promising to construct an applicable damage constitutive theory with firm physical basis.

This paper presents a quasi-micromechanical damage model for brittle materials weakened by a large number of distributed microcracks. The microcracking damage is characterized in terms of DMG and the scalar microcrack density, which function as an exact representation of the complex anisotropic damage and a key parameter in the proposed scheme for calculating the impacts of microcrack interaction, respectively. The concept of DMG, which may be formulated in the form of sets of microcrack orientations, has clear physical and geometrical meaning in the orientation space. The problem of damage evolution under complex loading can be solved with the aid of the computation rules in set theory. In addition, the effects of microcrack interaction on effective moduli can be calculated in this model as easily as the noninteracting or DCM. After considering various microcracking mechanisms, the formulated constitutive equations can be applied for the whole deformation process of brittle or quasi-brittle materials, including the stages of linear elasticity, non-linear damage hardening, post-peak stress drop and strain softening, though the physical process of damage localization and its implementation in constitutive relations are worth of further research.

In this paper, our attention is focused on the material behavior under tension, though the main idea can be extended to the case of compression by incorporating the more complex microcracking mechanisms of closed microcracks (Horii and Nemat-Nasser, 1985; Feng and Yu, 2002). In addition, further investigation to other microcrackrelated problems, such as microcrack coalescence and microcrack-induced scale effects, will certainly deepen our understanding to the deformation and failure behavior of brittle engineering materials.

\section{References}

Aboudi, J., Benveniste, Y., 1987. The effective moduli of cracked bodies in plane deformations. Eng. Fract. Mech. 26, 171-184.

Bristow, J.R., 1960. Microcracks and the static and dynamic elastic constants of anneals and heavily cold-worked metals. Br. J. Appl. Phys. 11, 81-85.

Budiansky, B., O'Connell, R.J., 1976. Elastic moduli of a cracked solid. Int. J. Solids Struct. 12, 81-97.

Carvalho, F.C.S., Labuz, J.F., 1996. Experiments on effective elastic modulus of two-dimensional solids with cracks and holes. Int. J. Solids Struct. 33, 4119-4130.

Feng, X.Q., 2001. On estimation methods for effective moduli of microcracked solids. Arch. Appl. Mech. 71, 537-548.

Feng, X.Q., Gross, D., 2000. Three-dimensional micromechanical model for quasi-brittle solids with residual strains under tension. Int. J. Damage Mech. 9, 79-112.

Feng, X.Q., Yu, S.W., 1995. Micromechanical modeling of tensile response of elastic-brittle materials. Int. J. Solids Struct. 32, 3359-3374.

Feng, X.Q., Yu, S.W., 2002. Damage Micromechanics of Quasi-Brittle Materials. Higher Education Press, Beijing.

Feng, X.Q., Li, J.Y., Yu, S.W., 2003. A simple method for calculating interaction of numerous microcracks and its applications. Int. J. Solids Struct. 40, 447-464.

Gurson, A.L., 1977. Continuum theory of ductile rupture by void nucleation and growth. Part I. Yield criterion and flow rules for porous ductile media. J. Eng. Mater. Technol. 99, $2-15$.

Hashin, Z., 1988. The differential scheme and its application to cracked materials. J. Mech. Phys. Solids 36, 719734.

Horii, H., Nemat-Nasser, S., 1983. Overall moduli of solids with microcracks, load-induced anisotropy. J. Mech. Phys. Solids 31, 155-171. 
Horii, H., Nemat-Nasser, S., 1985. Compression-induced microcrack growth in brittle solids: Axial splitting and shear failure. J. Geophy. Res. 90, 3105-3125.

Huang, Y., Hu, K.X., Chandra, A., 1994. A generalized selfconsistent mechanics method for microcracked solids. J. Mech. Phys. Solids 42, 1273-1291.

Ju, J.W., Lee, X., 1991. Micromechanical damage models for brittle materials. J. Eng. Mech. 117, 1495-1536.

Kachanov, M., 1994. Elastic solids with many cracks and related problems. Adv. Appl. Mech. 30, 259-445.

Kanninen, M.F., Popelar, C.P., 1985. Advanced Fracture Mechanics. Oxford University Press, London.

Krajcinovic, D., 1989. Damage mechanics. Mech. Mater. 8, 117-197.

Krajcinovic, D., 1997. Damage Mechanics. Elsevier, Amsterdam.

Krajcinovic, D., Fanella, D., 1986. A micro mechanical damage model for concrete. Eng. Fract. Mech. 25, 585-596.

Krajcinovic, D., Mastilovic, S., 1995. Some fundamental issues of damage mechanics. Mech. Mater. 21, 217-230.

Krajcinovic, D., Basista, M., Sumarac, D., 1991. Micromechanically inspired phenomenological damage model. J. Appl. Mech. 58, 305-310.

Lacy, T.E., McDowell, D.L., Willice, P.A., Talreja, R., 1997. On representation of damage evolution in continuum damage mechanics. Int. J. Damage Mech. 6, 62-95.
Lawn, B.R., Marshall, D.B., 1998. Nonlinear stress-strain curves for solids containing closed cracks with friction. J. Mech. Phys. Solids 46, 85-113.

Najar, J., 1994. Brittle residual strain and continuum damage at variable uniaxial loading. Int. J. Damage Mech. 3, 260276.

Nemat-Nasser, S., Hori, M., 1993. Micromechanics, Overall Properties of Heterogeneous Materials. North-Holland, Amsterdam.

Ortiz, M., 1985. A constitutive theory for the inelastic behavior of concrete. Mech. Mater. 4, 67-93.

Shao, J.F., Rudnicki, J.W., 2000. A microcrack-based continuous damage model for brittle geomaterials. Mech. Mater. 32, 607-619.

Sneddon, I.N., 1951. Fourier Transformation. McGraw-Hill, New York.

Swoboda, G., Yang, Q., 1999. An energy-based damage model of geomaterials. Int. J. Solids Struct. 36, 1719-1755.

Yu, S.W., Feng, X.Q., 1995. A micromechanics-based model for microcrack-weakened brittle solids. Mech. Mater. 20, 59-76.

Zheng, Q.S., Du, D.X., 2001. An explicit and universally applicable estimate for the effective properties of multiphase composites which account for inclusion distribution. J. Mech. Phys. Solids 49, 2765-2788. 